\title{
Second order controllability conditions for the controllability of control systems with drift
}

\section{Antonio Marigonda*}

Università degli Studi di Padova

E-mail: amarigo@math.unipd.it

The problem of controllability, i.e. the existence of controls steering a suitable neighborhood of the target set $S$ to the target itself, is crucial in Control Theory. In this presentation we will show a generalization of some classical sufficient conditions to nonlinear control systems with drift, with a quite general target. The condition will be also used to study regularity properties for the minimal time function.

* Speaker. 


\section{Preliminars and Notation}

Let $S \subseteq \mathbb{R}^{n}$ be closed. We denote, for $x \in \mathbb{R}^{n}$,

$$
\begin{aligned}
d_{S}(x) & =\min \{\|y-x\|: y \in S\} & & \text { (the distance of } x \text { from } S \text { ) } \\
\pi_{S}(x) & =\left\{y \in S:\|y-x\|=d_{S}(x)\right\} & & \text { (the projections of } x \text { onto } S \text { ) } \\
B(S, \rho) & =\left\{y \in \mathbb{R}^{n}: d_{S}(y) \leq \rho\right\} & &
\end{aligned}
$$

Suppose $f: \mathbb{R}^{n} \rightarrow \mathbb{R} \cup\{+\infty\}$ is lower semicontinuous, and epi $(f):=\{(x, \xi): \xi \geq f(x)\}$ and $\operatorname{dom}(f)=\left\{x \in \mathbb{R}^{n}: f(x)<+\infty\right\}$ are its epigraph and (effective) domain, respectively. Let $x \in$ $\operatorname{dom}(f)$. A vector $\zeta \in \mathbb{R}^{n}$ belongs to the proximal superdifferential of $f$ at $x$ (notated by $\zeta \in \partial^{P} f(x)$ if there exist $\sigma, \eta>0$ such that

$$
f(y) \leq f(x)+\langle\zeta, y-x\rangle+\sigma\|y-x\|^{2} \quad \text { for all } y \in B(x, \eta) .
$$

The proximal superdifferential of the distance function $d_{S}$ is always nonempty at all $x \notin S$.

We consider the control system: $\dot{y}=f(y, u), u \in \mathcal{U}$ where $\mathcal{U}$ is a compact convex subset of $\mathbb{R}^{m}, S$ is a closed subset of $\mathbb{R}^{n}$ called the target set, $f$ is Lipschitz continuous in $x$ in $\mathbb{R}^{n} \backslash S$, uniformly in $u$. In particular, we refer to the affine case, if $f$ takes the special form:

$$
f(x, u)=g_{0}(x)+\sum_{i=1}^{m} u_{i} g_{i}(x)
$$

where $u_{1}, . ., u_{m} \in[-1,1]$ and $g_{0}, g_{1}, \ldots, g_{m}$ are vector fields of $\mathbb{R}^{n}$ with some smoothness.

We denote by $\mathcal{F}$ the family of vector fields associated to $\dot{y}=f(y, u)$, i.e. for every $x \in \mathbb{R}^{n}$, we have $\mathcal{F}(x):=\{f(x, u): u \in \mathcal{U}\}$.

We denote by $y^{x, u}(t)$ the solution of the system starting from point $x$ obtained using the control $u(\cdot)$. For fixed $x \notin S$, the minimal time $T(x)$ to reach $S$ from $x$ is defined by

$$
T(x):=\inf \left\{T \geq 0: \exists u(\cdot) \text { such that } y^{x, u}(T) \in S\right\} .
$$

When the set of controls $u(\cdot)$ steering $x$ to $S$ is empty, then $T(x)=+\infty$. For $r>0$, let

$$
\mathcal{R}=\left\{x \in \mathbb{R}^{n}: T(x)<+\infty\right\}, \quad \mathcal{R}_{\delta}=(\mathcal{R} \backslash S) \cap B(S, \delta)
$$

\section{Sufficient conditions for controllability}

Consider the control system $\dot{x}=f(x, u), u \in \mathcal{U}$ set of controls with a smooth target $S$. Assume that there exist positive constants $\delta, \mu>0$ such that for all $x \in B(S, \delta) \backslash S$ we have:

$$
\min _{u \in \mathcal{U}}\left\langle D d_{S}(x), f(x, u)\right\rangle \leq-\mu \quad \text { (Petrov condition) }
$$

Then the system is controllable in $B(S, \delta)$. From a geometrical point of view, the condition states that at every point there exists an admissible control such that the corresponding trajectory points toward the target together with uniformity in the angle between the field and the gradient of the distance which prevents vanishing of the scalar product. This is a very strong condition and it can be proved that it is equivalent to Lipschitz continuity of the minimal time function $T$. See also [3], 
and [7].

In the case of linear systems $\dot{x}=A x+B u, A \in \operatorname{Mat}_{n \times n}(\mathbb{R}), B \in \operatorname{Mat}_{n \times m}(\mathbb{R}), u \in[-1,1]^{m}$, with target $S=\{0\}$ the Kalman rank condition gives sufficient condition for controllability and imply Hölder continuity of $T$. Let $j$ be the minimum positive integer such that the matrix:

$$
\left(B A B \ldots A^{j} B\right)
$$

has full rank. Then $T$ is $\left(\frac{1}{1+j}\right)$-Hölder continuous.

This is related to some properties of the Lie algebra generated by the family of vector fields associated to the system (see [6]). There are also some nonlinear version of this result concerning the linearization of the system near an equilibrium point (see [2]) where the target is the equilibrium point.

In [1] there is a condition for Hölder continuity of $T$ in the case of nonlinear symmetric systems $\dot{x}=\sum u_{i} g_{i}(x)$ for a smooth target. The condition requires that if at a point $\bar{x} \in \partial S$ Petrov condition does not hold, there exists a vector field $F(\bar{x})$ generated by bracket operations from the vector fields of the family $\mathcal{F}:=\{f(\cdot, u): u \in \mathcal{U}\}$ associated to the system such that:

$$
\langle F(\bar{x}), v(\bar{x})\rangle<0
$$

where $v(\bar{x})$ is the normal unit vector to the target $S$ at $\bar{x}$.

This condition can be viewed as a Petrov condition of higher order, and in fact it leads to Hölder continuity of $T$ and no longer to Lipschitz continuity, where the exponent of the modulus of continuity depends from the number of Lie brackets which are involved.

Example: $\operatorname{In} \mathbb{R}^{3}$ consider the system $\dot{x}=X_{0}+u_{1} X_{1}+u_{2} X_{2}$, where

$$
X_{0}=\frac{1}{12}(-y, x, 0), \quad X_{1}=(x z, y z, 0), \quad X_{2}=(0,0,1)
$$

and define as target set the cylinder $S:=\left\{(x, y, z) \in \mathbb{R}^{3}: x^{2}+y^{2} \leq 1\right\}$. The vector fields are $C^{\infty}$ in $\mathbb{R}^{3}$. We have for every $x \notin S$ :

$$
D d_{S}(x)=\frac{(x, y, 0)}{\sqrt{x^{2}+y^{2}}}
$$

and

$$
\left\langle D d_{S}, X_{0}+u_{1} X_{1}+u_{2} X_{2}\right\rangle(x, y, z)=u_{1} z \sqrt{x^{2}+y^{2}} .
$$

So Petrov condition fails in the plane $z=0$. The system is not symmetric since we have a nontrivial drift $X_{0}$, but $\left[X_{1}, X_{2}\right](x, y, z)=(x, y, 0)$. This case will be covered by Theorem 3.2, since the trajectory given by Lemma 3.2 approaches uniformly to the target.

\section{The main result}

In order to obtain estimates for the minimum time function in terms of the distance from the target $S$, we will use a sort of expansion of distance along the trajectories of the system in a neighborhood of the considered point. These expansion will be crucial for our analysis. Indeed, if 
the target is sufficiently smooth (e.g. $C^{2}$ ), we may consider the derivatives of $d_{S}$, but, in general, we can consider weaker assumption on $S$ (for example condition ensuring the semiconcavity of $d_{S}$ ). The smoother is the target, the finer will be the estimates. Now, in the following two lemmas, we consider two particular cases of affine systems with drift, and we write the expansion of particular trajectories generated by using some switchings of the control.

Using the exponential notation, introduced by A. Agrachev and R. Gamkrelidze (for further details we refer to [4] and references therein), we will denote by $x e^{t X}$ the point $y(t)=\exp (t X)(x)$ where $\exp (t X)(x)$ is the solution at time $t$ of the following Cauchy problem:

$$
\dot{y}=X(y), \quad y(0)=x \in \Omega .
$$

Lemma 3.1. Let $f, g$ be $C^{\infty}$ vector fields on $\mathbb{R}^{n}, \bar{x} \in \mathbb{R}^{n}, V_{\bar{x}}$ be an open bounded neighborhood of $\bar{x}$, and let $u \in \mathbb{R}$. For every $x \in V_{\bar{x}}$, set:

$$
\phi_{x}(t)=x e^{t(f+u g)} e^{t(f-u g)}
$$

Then it holds: $\phi_{x}(t)=x+2 t f(x)+\frac{t^{2}}{2}(D f(x) f(x)+u[f, g](x))+o\left(t^{2}\right)$, where $\left|o\left(t^{2}\right)\right| \leq L t^{3}$, with $L>0$ positive constant which does not depend on $x \in V_{\bar{x}}$.

Proof. Follows by direct computations.

Lemma 3.2. Let $f, g_{1}, g_{2}$ be $C^{\infty}$ vector fields on $\mathbb{R}^{n}, \bar{x} \in \mathbb{R}^{n}, V_{\bar{x}}$ be an open bounded neighborhood of $\bar{x}$. For every $x \in V_{\bar{x}}$, set:

$$
\phi_{x}(t)=x e^{t\left(f+g_{1}\right)} e^{t\left(f+g_{2}\right)} e^{t\left(f-g_{1}\right)} e^{t\left(f-g_{2}\right)} e^{t\left(f-g_{1}\right)} e^{t\left(f-g_{2}\right)} e^{t\left(f+g_{1}\right)} e^{t\left(f+g_{2}\right)}
$$

Then it holds: $\phi_{x}(t)=x e^{8 t f+2 t^{2}\left[g_{1}, g_{2}\right]}+o\left(t^{2}\right)$ where $\left|o\left(t^{2}\right)\right| \leq L t^{3}$, with $L>0$ a positive constant which does not depend on $x \in V_{\bar{x}}$.

Proof. See [4].

The following theorem provides a sufficient local condition to have continuity and some regularity estimates for the minimum time $T(\cdot)$ for a non linear system $\dot{x}=f(x, u), u \in \mathcal{U}$. Assume that the target set $S$ is of class $C^{2}$. We are interested in the expansion of the composition $d_{S}\left(y_{x}(t)\right)$ around $t=0$, where $y_{x}(t)$ is a generic trajectory of the system for which we know an expansion up to order two:

$$
y_{x}(t)=x+t \cdot v_{1}^{x}+\frac{t^{2}}{2} \cdot v_{2}^{x}+o_{x}\left(t^{2}\right)
$$

In this case:

$$
\begin{aligned}
d_{S}\left(y^{x, u}(t)\right)= & d_{S}(x)+t\left\langle D d_{S}(x), v_{1}^{x}\right\rangle+ \\
& +\frac{t^{2}}{2}\left(\left\langle\left\langle D^{2} d_{S}(x), v_{1}^{x}\right\rangle, v_{1}^{x}\right\rangle+\left\langle D d_{S}(x), v_{2}^{x}\right\rangle\right)+o_{x}\left(t^{2}\right) .
\end{aligned}
$$

In order to reach the target, we require two conditions:

1. the trajectory $y^{x, u}(t)$ for $t \geq 0$ small enough must approach the target, and this is implied by:

$$
\left\langle D d_{S}(x), v_{1}^{x}\right\rangle \leq 0, \quad\left\langle\left\langle D^{2} d_{S}(x), v_{1}^{x}\right\rangle, v_{1}^{x}\right\rangle+\left\langle D d_{S}(x), v_{2}^{x}\right\rangle<0 .
$$


2. we can follow the trajectory $y_{x}(t)$ for a certain time until the above condition are satisfied. Suppose we have reached a point $x_{1}$. We are approaching the target by (1). Then we choose another trajectory and restart form $x_{1}$ following the new approaching trajectory $y_{x_{1}}(t)$ and so on. In order to reach the target in a finite amount of time, we have to require that at each step the rate of approach is sufficiently large. This will be done by an integral estimate.

Theorem 3.1 (The uniform case) Consider the system $\dot{x}=f(x, u), u \in \mathcal{U}$. Suppose that the target $S$ is such that $d_{S}(\cdot)$ is semiconcave on $\mathbb{R}^{n} \backslash \operatorname{int}(S)$ with constant $K$, and assume that $\partial S$ is compact. Let $M=1 /\left(4 L+14 K L^{2}\right) \wedge 1 /\left(2 L+12 K L^{2}\right)$. Let $\delta>0$ and $\mu \geq 0, \eta>0$ be constant. We define for every $x \in B(S, \delta): T_{1}^{x}=M \mu \wedge 2 d_{S}(x) / \mu, \quad T_{2}^{x}=M \eta \wedge \sqrt{2 d_{S}(x) / \eta}$.

Let $V=B(S, \delta)$, assume that the following holds:

(S1) for every $x \in V$ there exists an admissible trajectory $y_{x}(\cdot)$ such that:

$$
y_{x}(t)=x+t \cdot v_{1}^{x}+\frac{t^{2}}{2} \cdot v_{2}^{x}+o_{x}\left(t^{2}\right)
$$

with $\left|o_{x}\left(t^{2}\right)\right| \leq L t^{3}$. and for this trajectory it holds either:

a) if $\mu>0, \exists \zeta_{x} \in \partial^{P} d_{S}(x):\left\langle\zeta_{x}, v_{1}^{x}\right\rangle \leq-\mu, \quad y_{x}\left(T_{1}^{x}\right) \in V$

or

$$
\begin{aligned}
& \text { b) } \exists \zeta_{x} \in \partial^{P} d_{S}(x):\left\langle\zeta_{x}, v_{1}^{x}\right\rangle \leq 0 \\
& \quad \text { and }\left\langle\zeta_{x}, v_{2}^{x}\right\rangle+2 K\left|v_{1}^{x}\right|^{2} \leq-2 \eta, y_{x}\left(T_{2}^{x}\right) \in V
\end{aligned}
$$

(S2) for every $x \in V$ we have $\left|v_{1}^{x}\right|+\left|v_{2}^{x}\right| \leq L$

Then $\mathcal{R} \supseteq B(S, \delta), T$ is continuous on $\mathcal{R}$ and we have $T(x) \leq \tilde{T}(x)$ for every $x \in B(S, \delta)$. In particular, if we can choose $\mu(\rho)=C_{1} \rho^{\alpha_{1}}$ and $\eta(\rho)=C_{2} \rho^{\alpha_{2}}$, we have that $T$ is Hölder continuous of exponent $\alpha=\alpha\left(\alpha_{1}, \alpha_{2}\right)$.

Proof.We give here a sketch of the proof, see [4] for the complete proof.

1. We use the superdifferential inequality 1.1 for the distance function along the trajectory.

2. Conditions $(S 1)$ ensures us that for a fixed amount of time $\bar{t}$ the trajectory is approaching the target and in the final point, condition $(S 1)$ still holds.

3. We give and estimate for $\bar{t}$ and for the rate of approach to the target.

4. We construct a sequence of points $x_{i}$ and times $t_{i}$ concatenating such trajectories and exploiting conditions $(S 1)$.

5. The sequence converges to the target in a finite amount of time. The minimum time $T$ is bounded from above by a continuous function of the distance $d_{S}$ and this gives the continuity.

Now we give a more general statement that allows to take nonconstant $\mu=\mu\left(d_{S}(x)\right), \eta=\eta\left(d_{S}(x)\right)$, in this case there is an integral condition ensuring convergence to the target in a finite time, and giving continuity on $\mathcal{R}_{\mathbb{S}}$ even if $\mathcal{R}$ is not a neighborhood of the target. 
Theorem 3.2. Consider the system $\dot{x}=f(x, u), u \in \mathcal{U}$. Suppose that the target $S$ satisfies an internal sphere condition with uniform radius and let $K$ be the (global) constant of semiconcavity of $d_{S}(\cdot)$ on $\mathbb{R}^{n} \backslash \operatorname{int}(S)$.

Let $M=1 /\left(4 L+14 K L^{2}\right) \wedge 1 /\left(2 L+12 K L^{2}\right)$. Let $\delta>0$ and $\mu, \eta:[0, \delta] \rightarrow \mathbb{R}^{+}$be two continuous, nondecreasing functions, with $\mu(\rho)>0$ and $\eta(\rho)>0$ when $\rho>0$, satisfying

$$
\int_{0}^{\delta}\left(\frac{1}{\mu(\rho)}+\frac{1}{M \eta^{2}(\rho)}\right) d \rho<+\infty, \quad \int_{0}^{\delta}\left(\frac{1}{\mu(\rho)}+\frac{1}{\sqrt{\rho \eta(\rho)}}\right) d \rho<+\infty .
$$

We define for every $x \in B(S, \delta)$ :

$$
T_{1}^{x}=M \mu\left(d_{S}(x)\right) \wedge 2 d_{S}(x) / \mu\left(d_{S}(x)\right), \quad T_{2}^{x}=M \eta\left(d_{S}(x)\right) \wedge \sqrt{2 d_{S}(x) / \eta\left(d_{S}(x)\right.},
$$

Let $V$ a subset of $B(S, \delta)$ such that the following holds:

(S1) for every $x \in V$ there exists an admissible trajectory $y_{x}(\cdot)$ such that:

$$
y_{x}(t)=x+t \cdot v_{1}^{x}+\frac{t^{2}}{2} \cdot v_{2}^{x}+o_{x}\left(t^{2}\right)
$$

with $\left|o_{x}\left(t^{2}\right)\right| \leq L t^{3}$. and for this trajectory it holds either

$$
\exists \zeta_{x} \in \partial^{P} d_{S}(x):\left\langle\zeta_{x}, v_{1}^{x}\right\rangle \leq-\mu\left(d_{S}(x)\right), \quad y_{x}\left(T_{1}^{x}\right) \in V
$$

or

$$
\exists \zeta_{x} \in \partial^{P} d_{S}(x):\left\langle\zeta_{x}, v_{1}^{x}\right\rangle \leq 0 \text { and }\left\langle\zeta_{x}, v_{2}^{x}\right\rangle+2 K\left|v_{1}^{x}\right|^{2} \leq-2 \eta\left(d_{S}(x)\right), y_{x}\left(T_{2}^{x}\right) \in V
$$

(S2) for every $x \in V$ we have $\left|v_{1}^{x}\right|+\left|v_{2}^{x}\right| \leq L$

Then there exists a continuous function $\tilde{T}$ such that $\tilde{T}(0)=0$ and $T(x) \leq \tilde{T}\left(d_{S}(x)\right)$ for every $x \in V$. In particular if conditions (S1) and (S2) hold for every $x \in \mathcal{R}_{\delta}$ we have that $T$ is continuous on $\mathcal{R}_{\delta} \cup S$. Moreover if ( $\left.S 1\right)$ and (S2) hold for every $x \in B(S, \delta)$ we have that $\mathcal{R} \supseteq B(S, \delta)$ and $T$ is continuous on $\mathcal{R}$.

Corollary 3.1. In the same hypothesis of Theorem 3.2. assume that $V=R_{\delta}$ and there exist an optimal control $u_{x}$ for $x$ and a neighborhood $V_{x}$ of $x$ depending on $x$ and $u_{x}$ such that $y^{z, u_{x}}(T(x)) \in \mathcal{R}$ for all $z \in V_{x} \cap V$. Then we have continuity of $T$ on $R \cap B(S, \delta)$ and we can estimate the modulus of continuity of $T$ with $\tilde{T}$. Moreover an estimate $\omega_{T}(\rho)=C \rho^{\alpha}$, which leads to Hölder continuity (see [1], [4]), can be obtained, for example, if in the Theorem 3.2 we can choose $\mu(\rho)=C_{1} \rho^{\alpha_{1}}$ and $\eta(\rho)=C_{2} \rho^{\alpha_{2}}$.

Proof. The proofs follows the same outline of the previous case. The difference is given by the fact that now at each step the rate of approach decreases, so integral condition plays a key role to have convergence in finite time. See [4] for details.

Remarks:

1. The condition on semiconcavity of $d_{S}$ can be satisfied requiring that $S$ satisfies an internal sphere 
condition with uniform radius (see [3])

2. In the case of smooth target, the term $D^{2} d_{S}$ is related to the curvature. In particular, targets with negative curvature can be approached better that others.

Example: The following example shows another situation where neither vector fields or Lie bracket can help, but by geometrical properties of the (smooth) target, such as negative curvature, we can construct a trajectory approaching the target itself. The system in $\mathbb{R}^{2}$ is $\dot{z}=X_{0}(z)+u X_{1}(z)$ where $X_{0}(z)=\left(0,-x^{2}\right), X_{1}=(1,0),|u| \leq 1$, and the target is:

$$
S:=\{|x| \geq 2, y \leq 1\} \cup B((2,0), 1) \cup B((-2,0), 1) \cup\{y \leq 0\} \backslash B((0,0), 1) .
$$

We focus our attention on a small neighborhood of the target $B(S, \delta), \delta>0$. The critical set is the $y$-axis $\{x=0\} \cap B(S, \delta)$, where $X_{0}$ vanishes and $\left\langle X_{1}, D d_{S}(0, y)\right\rangle=0$. Here, the trajectory requested by Theorem 3.2 is simply the one given by choosing $u= \pm 1$. We refer to [4] for a complete study of this case.

\section{Conclusions and open problems}

The given condition generalizes both Petrov condition (at first order) and Lie bracket condition for symmetric systems (at second order) and Kalman rank condition at second order. The results of [5], considering as target $C^{1}$-manifolds possibly with border (obtained with PDE methods), are still not fully covered.

Conditions at higher order encounter some technical problems due to the fact that it cannot be provided easily an expansion like the one contained in Lemma 3.2. Indeed at order higher than 2 the presence of mixed terms involving brackets of $f, g$ requires additional restrictions on vector fields.

\section{References}

[1] M. Bardi, I. Capuzzo-Dolcetta, Optimal Control and Viscosity Solutions of Hamilton-Jacobi-Bellman Equations, Birkhäuser, Boston (1997)

[2] R.M. Bianchini, G. Stefani, Sufficient conditions of local controllability, in Proc. 25th IEEE Conference on Decision and Control, 1986, pp. 967-970 SIAM J. Control and Optimization, Vol. 31, No. 4, pp. 900-927, July 2003

[3] P. Cannarsa, C. Sinestrari, Convexity properties of the minimum time function, Calc. Var. 3 (1995), 273-298.

[4] A. Marigonda, Second order conditions for the controllability of nonlinear systems with drift, preprint.

[5] P. Soravia, Hölder continuity of the minimum time tunction for $C^{1}$-manifold targets, Journal of optimization theory and applications, Vol 75, No. 2, November 1992

[6] V. Jurdjevic, Geometric control theory, Cambridge University Press, 1997

[7] V. Veliov, Lipschitz continuity of the value function in optimal control, J. Optim. Theory Appl. 94 (1997), 335-363. 